\section{Reply to letters regarding clinical features of chromosome 22q11 deletion}

One of the difficulties we face when we see anomalies/medical problems in people with a $22 \mathrm{q} 11$ deletion is deciding whether or not these are directly attributable to the microdeletion or are found by chance association. With what frequency does a second disorder need to occur before it can be said to be associated with the first?

Dean et al commented on the occurrence of craniosynostosis in 22q11 deletion patients. They reported an infant with a 22q11 deletion who had bilateral coronal and sagittal craniosynostosis. Subsequently this child was found to have a de novo P250R FGFR3 mutation. It is more likely that the craniosynostosis in this infant was the result of the FGFR3 mutation rather than the 22q11 deletion. However, the $5 / 548(0.91 \%)$ reported in our series exceeds the highest population estimate they give. They state that the birth incidence of craniosynostosis varies between $1 / 3225(0.03 \%)$ and $1 / 735(0.13 \%)$ live births, with the upper $95 \%$ confidence interval being $1 / 518(0.19 \%)$. However, we will proceed to obtain further clinical information on the five patients with craniosynostosis and undertake FGFR3 mutation analysis as they suggest. This should help to clarify whether craniosynostosis is a feature associated with $22 \mathrm{q} 11$ deletion.

In contrast, Di Rocco et al comment on the absence of polyarthropathy/juvenile rheumatoid arthritis (JRA) in association with the $22 \mathrm{q} 11$ deletion in our series. Their own experience of $3 / 80(3.75 \%)$ children at the Children's Hospital of Philadelphia having JRA gives an incidence of 50 times that of the general population. There were no reports of JRA/polyarthropathy in our series and it is unlikely that significant arthropathy would have been omitted from questionnaires. If the incidence is $3.75 \%$ we would have expected approximately 20 subjects in our series to have JRA. One of the strengths of our paper is the large number of patients reviewed. We hoped this study would clarify the incidence of uncommon features which may be overrepresented in published reports.

Di Rocco et al also comment that other autoimmune conditions have been observed in chromosome 22q11 deletion syndrome. In our study, $8 / 558$ (1.4\%) patients had autoimmune problems. Four patients had hypothyroidism; two were diagnosed in adult life, one at 5 years, and one at 12 years. This last patient had antithyroid antibodies. Interestingly, the patient's mother also had hypothyroidism, but did not have a 22q11 deletion. One patient had hyperthyroidism secondary to Hashimoto's thyroiditis aged 16 years. Two patients already previously reported had idiopathic thrombocytopenia and one patient had Raynaud's phenomena with positive anticardiolipin autoantibodies. A large proportion of the cases in our series are still children. Autoimmune phenomena may be one of the later complications of the disorder, indicating the importance of following the natural history of the disorder in a series of patients.

Hunter asks for further information about the ascertainment of the patients in the series. For the vast majority, the diagnosis was made by a paediatrician or in a general genetics clinic: 188 (34\%) were referred by cardiologists, $39(7 \%)$ through cleft palate clinics, and four by immunologists. It is difficult to disentangle whether the fact that 188 were referred by cardiologists is biased ascertainment or simply reflects the fact that many of these children will present to a cardiologist. However, it is true to say that as our experience of the condition increases, we are making the diagnosis in children with fewer hard signs. The only way of being certain that there is no ascertainment bias would be to screen newborns to identify a cohort of children with deletions.

In conclusion, we feel that the series we have described is the least biased available, while recognising that it may have underestimated adult onset complications. As we become better at recognising the condition, it may become apparent that these cases still represent the more severe end of the spectrum.

Erratum. The reference Dean JCS, Cole GF, Appleton RE, Burn J, Roberts SA, Donnai D. Cranial hemihypertrophy and neurodevelopmental prognosis. F Med Genet 1990;27:160-4 should have been De Silva D, Duffty P, Booth P, Auchterlonie I, Morrison $\mathrm{N}$, Dean JCS. Family studies in chromosome 22q11 deletion: further demonstration of phenotypic heterogeneity. Clin Dysmorphol 1995;4:294-303.

A RYAN

J A GOODSHIP D I WILSON

ON BEHALF OF THE EUROPEAN CONSORTIUM

Department of Human Genetics, University of Newcastle upon Tyne, Newcastle upon Tyne, UK

\section{Absence of a del(22q11) in a patient with the $3 \mathrm{C}$ (craniocerebellocardiac) syndrome}

We read with interest the letter by Butler and Mowrey ${ }^{1}$ concerning the hypothesis that the 3C syndrome could be associated with a deletion of 22q11.2. In 1994, we had the opportunity to see a 10 day old female newborn who we then diagnosed as having the 3C syndrome. The case report was published in $1995 .^{2}$

We were able to see her again at 3 years 6 months of age and we performed molecular cytogenetic testing looking for a deletion of 22q11.2. The proband had moderate developmental delay, short stature with macrocephaly (height was $85 \mathrm{~cm},<5$ th centile weight was $10.75 \mathrm{~kg}$, <5th centile, and OFC was $53.5 \mathrm{~cm},>97$ th centile). Surgery for glaucoma, atrial septal defect, pulmonary stenosis, and a ventriculoperitoneal shun had been successful. She still had most of the dysmorphic features of the $3 \mathrm{C}$ syndrome (fig 1), including a bulging forehead, a prominent occiput, ocular hypertelorism (inner canthal distance $35 \mathrm{~mm},>+2 \mathrm{SD}$; outer canthal distance $98 \mathrm{~mm},+2 \mathrm{SD}$ ), epicanthus, and depressed nasal bridge. Immune function was normal both in the first year of life (serum immunoglobulins) and now (absolute number of $T$ lymphocytes, $T$ cell subsets, and serum immunoglobulins)

For the detection of the 22q11.2 deletion by fluorescent in situ hybridisation (FISH), a digoxigenin labelled locus specific probe, D22S75, which lies in the commonly deleted region, was used, in combination with a chromosome 22 control probe, D22S39, which is located at 22q13.3. FISH was performed on metaphase spreads from peripheral blood lymphocytes according to the manufacturer's recommendations (Oncor), but no microdeletion was visible.

The $3 \mathrm{C}$ syndrome is characterised by central nervous system, cardiac, and craniofacial anomalies. It is presumed to be autosomal recessive (MIM 220210 ${ }^{3}$ ) and 18 cases have been published, ${ }^{245}$ eight of them from seven families of Canadian native Indians.

Reviewing these 18 case reports, although there may be an overlap in the cardiac defects with the DiGeorge/velocardiofacial phenotype, ${ }^{\circ}$ the characteristic facies are remarkably different. The patient with a deletion of 22q11.2, ${ }^{7}$ who impressed Butler and Mowrey, ' has none of the dysmorphic features of the $3 \mathrm{C}$ syndrome. Mental retardation was present in all $3 \mathrm{C}$ patients, ${ }^{2}{ }^{45}$ absent in the patient ${ }^{7}$ commented on by Butler and Mowrey, ${ }^{1}$ and is reported as a feature of $40 \%$ of velocardiofacial ${ }^{8}$ and $77 \%$ of DiGeorge syndrome 9 patients. Palatal or pharyngeal abnormalities were described in $11 \%$ of $3 \mathrm{C},{ }^{2{ }^{5}} 98 \%$ of velocardiofacial, ${ }^{8}$ and $48 \%$ of DiGeorge' patients. The only immunodeficiency associated with the $3 \mathrm{C}$ syndrome was a humoral one ${ }^{10}$ and hypocalcaemia has never been reported.

In another case report, ${ }^{11}$ a deletion in $22 \mathrm{q} 11$ was found in a patient with the initial diagnosis of $3 \mathrm{C}$ syndrome. The presence of microcephaly described in this patient ${ }^{11}$ is quite unusual, as macrocephaly was a feature of 11 of the 18 reported cases of $3 \mathrm{C}$ syndrome. ${ }^{245} \mathrm{He}$ also had a multicystic and dysplastic kidney, and the only report of renal abnormalities in other 3C patients is a prenatal ultrasound diagnosis of bilateral hydronephrosis, which subsided leaving a unilateral dilated collecting system. ${ }^{12}$ Taking also into account the absence of cardiac anomaly, a feature of 16 of the 18 reported cases of $3 \mathrm{C}$ syndrome, ${ }^{245}$ and the facial features of the patient in the published picture, ${ }^{11}$ it seems to be a less characteristic case.

However, deletions of 22q11.2 have been detected in a heterogeneous variety of patients. Some cases do not have features included in the acronym CATCH 22 (Cardiac, Abnormal facies, Thymic hypoplasia, Cleft palate, Hypocalcaemia), such as laryn-

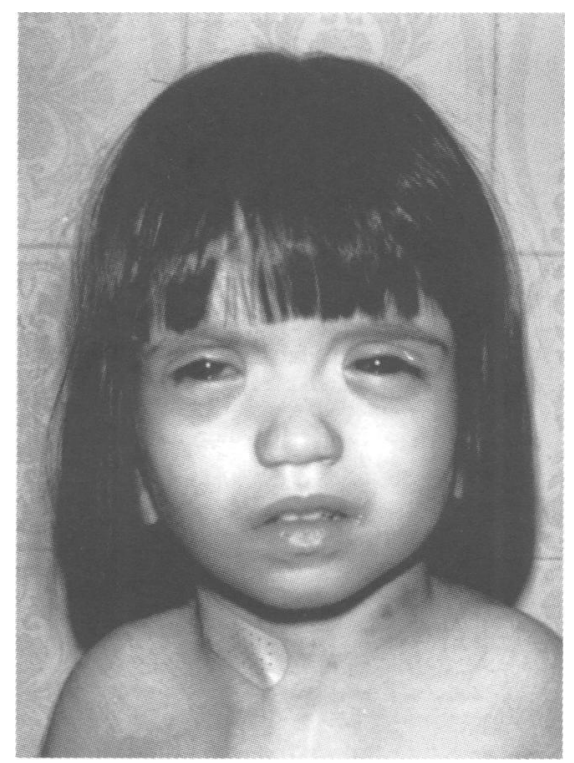

Figure 1 Patient with ocular hypertelorism, epicanthus, and depressed nasal bridge. 